\title{
PROTECTING JAVA COMPONENT INTEGRITY AGAINST TROJAN HORSE PROGRAMS
}

\author{
Marcel Winandy, Armin B. Cremers, Hanno Langweg and Adrian Spalka \\ Department of Computer Science III, University of Bonn \\ Roemerstrasse 164, D-53117 Bonn, Germany \\ Email: adrian@cs.uni-bonn.de
}

\begin{abstract}
We discuss how integrity of components of Java applications can be protected. Intrinsic and extrinsic protection mechanisms are employed. Capabilities of the Java application programming interface include permissions and signed JAR files. The strength of the methods depends on the operating system in which the Java virtual machine is embedded. Possible attacks by Trojan horse programs on component integrity are given and an approach to prevent or detect these is presented.
\end{abstract}

Key words: Java, component integrity, Trojan horse programs

\section{INTRODUCTION}

Java as a programming language has gained a widespread acceptance in various places. It is being used on servers, desktop machines or mobile devices alike. Some of the applications include security-sensitive services, e.g. cryptographic operations to ensure confidentiality or integrity of data.

In the design of the Java architecture security plays a notable part. Type safety and avoidance of pointer arithmetic are a great help in averting programming errors that may lead to security vulnerabilities. Access control mechanisms make certain that code can only access valuable resources it has been granted access rights for. In the context of security-sensitive applications integrity of the collaborating components is a key factor of concern.

On a desktop computer many programs may be installed and active at any given moment. Not all of them can be trusted, i.e. they may be Trojan

The original version of this chapter was revised: The copyright line was incorrect. This has been corrected. The Erratum to this chapter is available at DOI: 10.1007/978-0-387-35693-8_16 
horse programs harbouring a hidden and malicious functionality intended to work against the user's intentions. While no user would deliberately start a malicious program, the history of attacks based on viruses, worms, Trojan horses and other rogue programs convincingly demonstrates that an attacker can have various ways to start an attack on the user's personal computer. The Internet is also a perfect means for distributing such programs.

Component integrity means that a component's information will not be altered accidentally or maliciously. A violation of a component's integrity can have fatal consequences, especially if it is left undetected. In this paper we examine how Java assists application developers and users in ensuring component integrity for security-sensitive programs. This is part of a comprehensive approach to protect security-sensitive applications. We show that Java's security architecture relies on the underlying operating system. Secure execution of Java programs cannot be ensured if the operating system's integrity is violated.

The paper is organized as follows. An overview of the Java security architecture is given in section 2 . The following section provides an overview of previous and related work. A threat scenario is outlined in section 4 . We discuss Java permissions to preserve interface semantics in section 5 and show how code integrity can be maintained statically by signed JAR files in section 6. Dynamic mechanisms are also considered. Attacks on the execution environment are assessed in section 7. Our approach to counter attacks which involves a restricted execution environment is presented in section 8 and is followed by conclusions in section 9 .

\section{JAVA SECURITY ARCHITECTURE}

The Java security architecture consists mainly of code verifications to ensure type safety on loading of class files and access control based on permissions that can be defined in a security policy (Gong, 1999).

\subsection{Policy, permissions, and protection domains}

Individual access rights of a Java program are assigned by way of a policy object which represents the security policy of the Java virtual machine (JVM). The contents of the object are by default obtained from a policy file stored in the local file system. If no rules are specified, the JVM reverts to a sandbox model, i.e. local code is given full access to system resources, and code from remote network sources gets only restricted access. This holds for both Java applets and Java applications. 
The Policy class is an abstract class, i.e. a derived class has to be loaded by the JVM. Hence, custom implementations are possible, e.g. policies can be acquired from a database or a network server at the discretion of the developer. Basically speaking, the policy object is an access control matrix linking code sources to permissions. A code source is expressed as a URL, sometimes amended by code certificates.

Permissions represent access rights to resources. All permissions in Java are defined positively, i.e. access can only be granted but not denied explicitly. Permissions exist in a hierarchy, the abstract top-level class is java.security.Permission. Subclasses can be used to define (application) specific resources. For instance, java.io.FilePermission("/tmp/foo.txt", "read") specifies read access to the file /tmp/foo.txt. The permission AllPermission implies all other permissions. An object being assigned AllPermission possesses all rights in the JVM.

Permissions can also be assigned to protection domains instead of classes directly. A protection domain is a set of objects accessible by a principal, i.e. a user or a different software service. Every class and every object belongs to a distinct protection domain and inherits access rights of the domain. Code is assigned a protection domain by the Java execution environment. It is defined before the class can be used and the domain cannot be changed during runtime. System classes of the JVM belong to a special protection domain, the system domain. The system domain automatically has AllPermission access rights.

\subsection{Class loader}

Java classes are loaded by a class loader. They can be loaded just in time. The class loader mechanism facilitates the installation of software components during runtime.

When a class is to be loaded, the bytecode verifier of the JVM checks the integrity of the class' bytecode. The class loader determines the code's origin and examines the signature if there is one. According to the policy object the class is assigned a set of access rights and a class object is defined. Subsequently objects of the class can be instantiated.

Each class loader has a unique namespace. The type of a class is determined by the class name and the corresponding class loader. Classes loaded by different class loaders are treated as having different types by the JVM.

For additional checks custom class loaders can be implemented. Since class loaders are Java classes themselves they have to be loaded by another class loader. Thus a class loader hierarchy is established with the primordial 
class loader at its top. The primordial class loader is implemented natively and can not be accessed by Java code.

A class loader must not load the same class twice for the sake of type safety. However, it is possible to load the same class with different class loaders. Each class then has its own type as mentioned earlier.

\subsection{Security manager and access controller}

Upon access to a resource that is restrained by access rights the SecurityManager is consulted whether that requesting class has sufficient permissions. The security manager is not required to exist and can vary during runtime.

Newer Java versions delegate all requests to the AccessController which implements the standard access control algorithm. This class is declared final, i.e. there can be no derived and modified classes. Therefore the access controller is always present and cannot be altered during runtime. An application is supposed to call the checkPermission method of the access controller to determine if a certain class has a desired permission.

The access control algorithm allows an access to a resource if and only if every protection domain in the current execution context has the requested permission. Otherwise it throws an AccessControlException. Every method belongs to a class and every class belongs to a protection domain. Hence, all calling stack frames in the current execution context of an application are inspected. In some cases this may be too restrictive. By putting code in a doPrivileged () block evaluation is done independently of the caller. However, classes that are used inside the block must still have the necessary permissions.

\section{PREVIOUS AND RELATED WORK}

There exist several papers on Java's security architecture. Sterbenz (1996) criticizes in his paper that methods declared as 'native' gain full access to system resources, disregarding a custom security policy. Exceptions owing to a cancelled access to a resource can be caught by an application and never reach the user's conscience.

Iliadis et. al. (1998) discuss threads. Permissions of a thread are the intersection of all its callers' permissions. If a thread enters a protection domain with a richer set of permissions it maintains its original permissions. If a protection domain has fewer permissions, the thread is assigned this smaller set of permissions. 
Verification of type safety of the JVM is done by data flow analysis of the bytecode verifier (Goldberg, 1998) or by a formal specification of the class loader (Qian et. al., 2000). Wallach et. al. (1998) examine the "stack inspection" algorithm that checks the access control's execution context, and model it with a logic-based approach. Termination of the algorithm is shown and it is proven that access is never granted incorrectly. However, under certain circumstances access may be denied though it is acceptable under the security policy. This is not considered a security problem.

Protection of the internal state of an object is discussed in Gong's paper (1998). Access to an object can be protected by another object, called a "guard". A permission object is an example of a guard. Confidentiality is achieved by encryption of the object's attributes, integrity relies on digital signatures. The JSEF framework (Hauswirth et. al., 2000) extends Java to allow the specification of negative permissions, i.e. accesses can be denied explicitly. JAAS (Lai et. al., 1999) augments Java's code-based security model by user-orientated access controls. Malabarba et. al. (2000) modify the JVM in order to replace classes dynamically during runtime.

An approach to preserve interface semantics is given in the Kava Java extension (Welch et. al., 1999). Transformations of bytecode are used to add security checks when classes are loaded. Method calls, initialization, and state changes of objects are now controlled by Kava. Bindings define what is to be controlled. However, Kava has to be added to the trustworthy part of the JVM. Therefore, whenever there is an update of the JVM or the bytecode format an update of the Kava extension is necessary. Bindings are not protected by Kava and could be manipulated. A noteworthy advantage of the approach is that the security model can be developed and changed separately from the application. On certain occasions this may not be desired, though.

\section{THREAT SCENARIO}

We assume that we have a security-sensitive application as a Java program, e.g. to perform some cryptographic operation. The JVM and its execution environment are implemented correctly, i.e. there are no 'bugs' that make the system vulnerable. The user is trustworthy and receives the program via a trustworthy distribution channel. The client system is supposed to be trustworthy prior and during installation.

This assumption does not hold after installation. At that time, malicious code, i.e. Trojan horse programs, may exist on the system possibly owing to the installation of programs of dubious origin.

A Trojan horse program acting like an ordinary program can use the operating system, the JVM and their respective application programming 
interfaces. Only the intended semantics of the provided interfaces are considered. Likely attacks may aim at retrieving secret information, e.g. passwords or cryptographic keys. The attacker could use object methods that are intended to be called only by other objects.

Exchanging or manipulating components prior to launching the securitysensitive application is also a probable threat. This could then lead to a manin-the-middle kind of attack inside the application itself. A replaced class responsible for reading a file, for instance, could supply the caller with contents of a different file. In a cryptographic context the generation of random numbers could be tampered with in case doctored initialization values are provided.

\section{PRESERVATION OF INTERFACE SEMANTICS}

\subsection{Basic model of an application}

We give a Java application example. Let a class User be the main program. The User object calls two more classes: $B$ and $C . B$ provides a method to compute a value, and $C$ has a method to just write "hello" on the screen. These classes represent a generalization of a Java program with multiple components. Class $B$ could represent an encryption operation, and $C$ could represent a progress bar. A graphical illustration is given below.

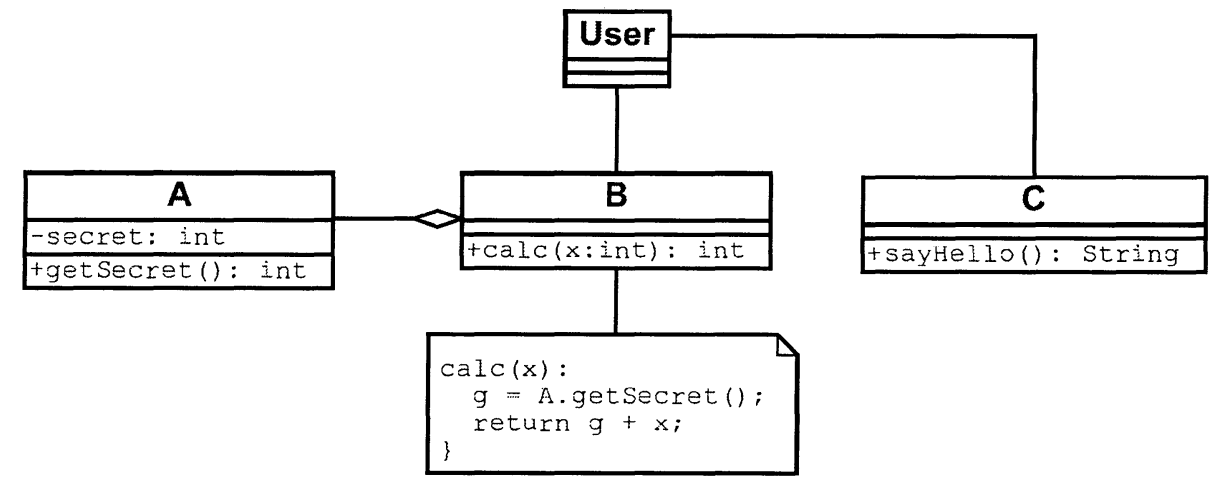

Fig. 1: Class diagram of the example

We assume that $B$ needs some information from an object $A$, e.g. a private encryption key. Only $B$ should be able to retrieve this information, $C$ should not. Merging $A$ into $B$ is not an acceptable solution since the application should remain divided into separate components. The following sequence diagram depicts the order in which the components interact. 


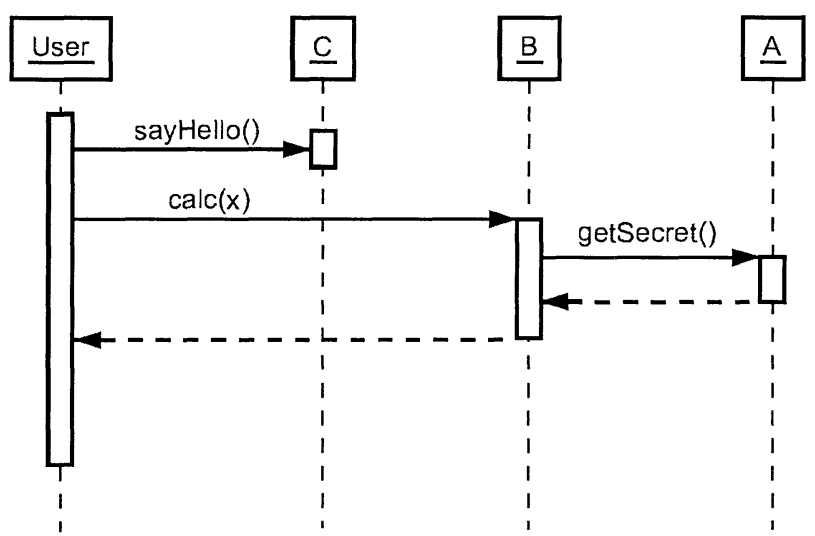

Fig. 2: Sequence diagram of the example

\subsection{Protection by JVM instances}

We now assume that an attacking Trojan horse program comes as a Java program accessing only the JVM application programming interface (API) and the Java system classes. If it creates own instances of class $A$, these get initial values. The desired secret is computed during runtime, possibly dependent on a password entered by the user. So it is not available if the class is newly instantiated by a malicious program. Even if $A$ was a Singleton class, i.e. there exists only one object of this class, an attack would not be possible. This owes to each Java program having its own JVM instance. Communication between different JVM instances is not allowed by default (Sun 2001, "System Goals").

A direct object to object communication between two JVM instances requires CORBA (Common Object Request Brokerage Architecture) or RMI (Remote Method Invocation). In CORBA an object registers with the object request broker and provides methods that can be called by other objects. RMI on the other hand transfers copies of objects. Such an object has to implement the java.rmi.Remote interface and thereby marks all its methods callable from every JVM. These 'remote objects' are required to register with the RMI Remote Object Registry before they can be accessed.

CORBA as well as RMI demand a registration. Objects that do not want to be accessed from other, possibly malicious, objects can skip registration and are henceforth inaccessible. However, in a distributed environment, this lack of functionality may not be desired. 


\subsection{Protection by permissions}

In this section we assume that a Trojan horse program is executed in the same address space as the user program, i.e. it is executed in the same JVM and has been loaded by the same class loader. This could be achieved by replacing a Java bytecode file with a modified version.

\subsubsection{Substitution of class $C$ by a Trojan horse program}

A class that is supposed not to access the secret value stored in $A$ is replaced with an altered version. This new class simulates $C$ 's behaviour and writes "hello" on the screen; in addition, it has a hidden functionality attempting to retrieve $A$ 's secret.

In general it is possible to access other objects' methods when their references are known. References become known by class methods that return such references, e.g. when using the Singleton class. Another way is to use the Reflection API to find objects in the same JVM. If a Trojan horse object has RuntimePermission("getClassLoader"), it can get a reference of its class loader.

By way of the class loader already loaded class objects can be discovered. This is done with findClass () and findLoadedClass (). Both methods are protected and therefore cannot be called directly by the attacker. Using loadClass (), however, with the desired class name as a parameter, the caller gets a reference to the class object. If the class had not already been loaded, it is loaded prior to returning the reference to the object. Hence, a Trojan horse object can retrieve references to class objects that are identified by their names.

The class object can be used to create new instances of the class. This does not pose additional problems since new instances only contain default values that do not have to be protected. In connection with the Singleton class, however, the retrieved reference points to an already existing object. By using getMethod (). invoke () class methods can be called. In our example, a Trojan object $C$ would call get Secret () of the existing class object $A$ and get the secret value.

As long as only class references are obtained in contrast to object references, no security problems arise. Application developers are urged to check whether calls of methods yield object references that could be exploited. 


\subsubsection{Counter measure}

To bypass a complete and tedious analysis of the source code to look for suspicious call sequences revealing object references, permissions offered by Java can be used.

In our example we create our own permission class, MyPermission, to access A's getSecret() method. Our class is derived from java.security.BasicPermission. A new named permission, e.g. "getSecret" is created and checked in the getSecret ( ) method. The new permission is assigned all classes that are supposed to access the secured method, i.e. $B$.

Apart from providing the secret value, $A$ has to check for the necessary permission:

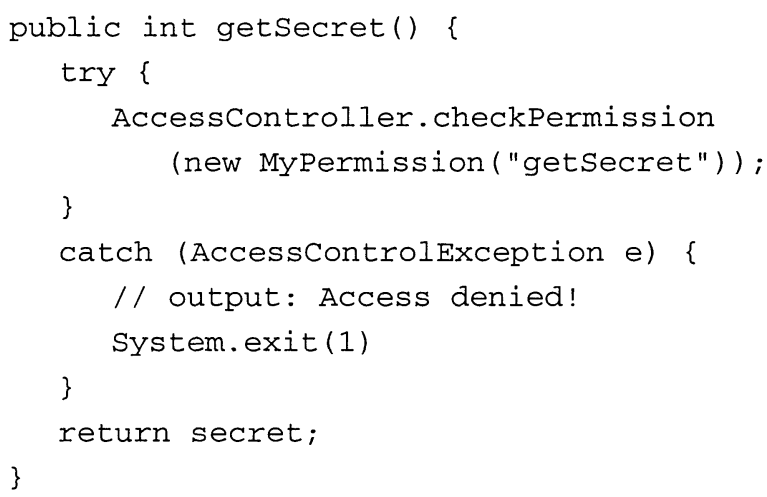

An exception should be handled immediately to prevent a Trojan horse from withholding it being reported to the user, concealing an attack's existence.

Assignment of access rights is done in a Policy file. In our example, A.getSecret () is called by a class $B$ object that had been called by a User object. This implies that User, $B$, and $A$ need an access right. Permissions can only be assigned to directories or JAR files. One way would be to group all three classes into a JAR file, and then assign the rights to this file. If the three classes need additional permissions that are not the same for each class, we would have to create different JAR files for the classes to be able to assign access rights correctly.

If the file my.policy contains the assignments of permissions, these permissions are applied by executing the JVM with java -Djava.security.policy=my.policy. It has to be ensured that the Policy file cannot be modified by an attacker. 
In case the calling chain $U s e r-B-A$ gets longer by introduction of additional components, the risk that a Trojan horse component gets inserted in the chain increases. So, it may not be desired to assign all classes in the chain access rights. This can be applied by using the doPrivileged() method of the Java AccessController. A.get Secret ( ) is called by $B$ in a doPrivileged() block. Then only $B$ (and $A$ ) need the getsecret permission. This follows the principle of least privilege assignment.

A Trojan horse component $C$ calling A.get Secret () now causes the AccessController to throw an exception because of a lacking permission in the Policy file.

\section{PRESERVATION OF COMPONENT INTEGRITY}

\subsection{Protection by signed JAR files}

In the preceding section we discussed how Java permissions help in preserving interface semantics against Trojan horse programs in the address space of the protected application. If it comes to the replacement of a component that has correct permissions set in the Policy file, the access control mechanisms of the JVM do no longer suffice.

\subsubsection{Substitution of class $A$ by a Trojan horse program}

Assume that class $A$ gets its secret information from an external source, e.g. a database. A Trojan horse program replacing class $A$ with a manipulated version would get this information and be able to transfer it to the attacker.

\subsubsection{Counter measure}

Java offers signing (and verification of signatures) of JAR files. In the Policy file permissions can be assigned to directories, JAR files, and also to signed code. JAR files can be signed by help of the jarsigner tool included in the Java development kit. This program uses cryptographic keys from the Keystore file that are generated with the keytool program. Signing is usually done with keys of 1024 bit length and the DSA-SHA-1 algorithm.

An application developer creates separate JAR files for each component to assign permissions on a least privilege basis. Then the JAR files are signed with the developer's private key. The public key used for verification 
is exported to a Keystore file. In the Policy file it is stated which Keystore file is to be used for verification. When distributing the program the JAR files are transferred together with the Policy and the Keystore files.

Policy and Keystore files have to be protected by external means of the execution environment, e.g. the operating system, against modification by an attacker. They are provided as parameters to the JVM upon launching the application.

A signed JAR file replaced with a manipulated version can be detected by the JVM because the signature of the new file will not be correct and thus can be denied execution.

Permission classes can also be signed. In the Policy file it can be stated that only signed permissions are assigned to protection domains. Permissions that are not correctly signed are not assigned to a protection domain.

\subsection{Dynamic defence mechanisms}

The methods presented so far allow a static check of classes. It is desired being able to verify that two objects communicating with each other are what they pretend to be. Up to JDK version 1.3.1, Java cannot replace classes during runtime. However, research in this direction has advanced (Malabarba et. al., 2000). Sun's JDK version 1.4.0 introduces the ability to substitute modified code in a running application through the debugger APIs (Sun, 2002a). It is shown in the rest of this section that Java does not provide suitable mechanisms to dynamically verify the integrity of components.

\subsubsection{Serializable interface}

With the Serializable interface objects can be written to and read from a stream. Hence, an object could be written to a byte stream and subjected to a SHA-1 algorithm. Sun (2002b) states that the class, the class signature and values of all non-transient and non-static fields of a class and all its parent classes are stored. This poses a problem. Values of object fields could change during runtime making a fixed hash value for identification unlikely. Another issue is adding a private method to a class, supposedly by a Trojan horse component. If this new private method is called within another existing class method the byte stream does not change leaving the modification undetected.

\subsubsection{Reflection API}

For every object getClass () retrieves the corresponding class object. It can be regarded the entry point of the Reflection API. Information about 
the object can be collected using java.lang.Class and java.lang.reflect. These mechanisms yield methods and fields of a class, implemented interfaces and associated packages.

A Trojan horse program could have added to or removed methods from a class, it could have changed existing method declarations or method modifiers. A couple of these modifications can be detected. getDeclaredMethods, getDeclaredConstructors, getDeclaredFields, and getDeclaredClasses are accessible by other classes for supervision purposes.

However, there are no methods to inspect a method body. A Trojan horse program could imitate a class altering its behaviour only by implementing a different method body. The Reflection API cannot be used to detect this then.

\subsubsection{Challenge-response protocols}

Another idea is to consider objects and object references nodes and edges in a (small) network. In networks challenge-response protocols are used to allow two nodes to identify each other prior to security-sensitive communication (Menezes et. al., 1997, chapter 10).

The basic design is as follows. Two nodes $\mathrm{A}$ and $\mathrm{B}$ want to identify each other. $\mathrm{B}$ sends a random value $\mathrm{x}$ to $\mathrm{A}$, called the challenge. A uses a secret function $\mathrm{s}$ to compute $\mathrm{s}(\mathrm{x})$, and sends $(\mathrm{s}(\mathrm{x}), \mathrm{y})$ to $\mathrm{B}$, called the response, where $\mathrm{y}$ is another random value, picked by $\mathrm{A}$. B also knows $\mathrm{s}$ and computes $\mathrm{s}(\mathrm{y})$ which is sent to $\mathrm{A}$. A and B can now verify with a certain possibility that they both know a common secret: $\mathrm{s}$. The values $\mathrm{x}$ and $\mathrm{y}$ have been chosen at random and a guess for $\mathrm{s}(\mathrm{x})$ or $\mathrm{s}(\mathrm{y})$ succeeds only with a low possibility.

Such a protocol is not suitable for the identification of Java objects. If we assume that an attacker replaces class $A$, and $A$ 's source code is known, then also the secret function $\mathrm{s}$ is known. Hence, the common secret is available to the adversary and cannot be used for identification.

\section{ATTACKS ON THE EXECUTION ENVIRONMENT}

Java provides protection mechanisms against direct attacks on an application. However, it depends on Policy and Keystore files that are not protected by the JVM itself. These files contain the definition of permissions and public keys for signature verification of JAR files. A modification of the files 
jeopardizes Java's security assumptions. These files have to be protected externally, e.g. by the operating system executing the JVM.

Attacks on the execution environment of the JVM include the replacement of program or system components, e.g. device drivers or code libraries. Another issue is a Trojan horse hooked between the JVM and the operating system intercepting and manipulating system calls. A Trojan horse program could replace the JVM or parts of it with altered versions. Thereby an attacker could gain knowledge about the internal state and data of a Java program. The execution could be manipulated at the attacker's choice.

Replacement of the JVM or parts of the operating system should not be possible or at least should not happen undetected. Java itself does not provide protection against this.

\section{MODULE STAMPING AND A RESTRICTED EXECUTION ENVIRONMENT}

Integrity of the Java application requires integrity of the application's code and its execution environment, i.e. the JVM and the operating system. The integrity of the used components can be checked upon launching the application. Since the application's code can be checked by the JVM we concentrate on verifying the integrity of the JVM and its execution environment.

All the modules that are necessary come in the form of files. These files are once subjected to a cryptographic one-way hash function. We call this process "stamping" and the resulting hash values "stamps". Before the application is launched, all the hash values are computed again and checked against the originally determined stamps. If the values do not match a modification has occurred and the integrity been violated.

To protect the stamps against manipulation a stamp of all stamps is calculated and stored in memory. A decent operating system protects the memory of an application against access by other applications and hence that value can reliably be used to verify the stamps' correctness. The integrity verification service is launched when the operating system is booted and is permanently running.

We have implemented this integrity verification service as a Windows 2000 service being executed permanently in the background. This service automatically determines modules, i.e. shared libraries and drivers, which are used by a process and then confirms their stamps. It is responsible for creating and maintaining stamps. It issues a warning to the user in case it detects an integrity violation. The user can request the service to check specific files like the Keystore and Policy files. 
To prevent manipulations of the service we have developed a restricted execution environment for the installation of new software requiring administrative privileges. We are able to allow the installation of untrustworthy programs without putting our integrity verification service at risk. Our service and the restricted execution environment employ special features of the Windows 2000 operating system. First results with the tool are very encouraging. However, giving a detailed implementation lies outside the scope of this paper.

\section{CONCLUSION}

We have examined the Java security architecture and how it can be applied to protect the integrity of Java programs. Mechanisms have been discussed to preserve interface semantics and signing of JAR files has been studied. Java relies on its surrounding execution environment to reliably provide its protection services.

Java has been considered with a focus on locally executed security-sensitive applications. The consequences of distributed applications that for instance employ the CORBA architecture remain to be scrutinized. However, the results shown in this paper can be generalized since permissions and code signing also work in distributed Java environments.

To maintain the integrity of the JVM and the necessary operating system components an integrity verification service has been designed and implemented for the Microsoft Windows 2000 platform. The findings can be transferred to other operating system platforms as well, e.g. Linux. Further work is necessary to analyse the specific capabilities and vulnerabilities of different platforms to assure a high level of resistance against attacks on the service.

\section{REFERENCES}

[1] Goldberg, A. (1998). "A Specification of Java Loading and Bytecode Verification". Proceedings of the 5th ACM Conference on Computer and Communications Security. pp:49-58.

[2] Gong, L. (1998). “Secure Java Class Loading”. IEEE Internet Computing 2(6):56-61.

[3] Gong, L. (1999). Inside Java 2 Platform Security. Addison-Wesley.

[4] Gong, L., and Dodda, S. (1999). "Security Assurance Efforts in Engineering Java 2 SE (JDK 1.2)". Proceedings of the 4th IEEE International Symposium on High-Assurance Systems Engineering.

[5] Gong, L., and Schemers, R. (1998). "Signing, Sealing, and Guarding Java Objects". Lecture Notes in Computer Science, Vol. 1419. pp:206-216. 
[6] Hauswirth, M., Kerer, C., and Kurmanowytsch, R. (2000). "A Secure Execution Framework for Java". Proceedings of the 7th ACM Conference on Computer and Communications Security. pp:43-52.

[7] Iliadis, J., Gritzalis, S., and Oikonomou, V. (1998). "Towards Secure Downloadable Executable Content: The JAVA Paradigm". Lecture Notes in Computer Science, Vol. 1516. pp:117-127.

[8] Kim, G.H., Spafford, E.H. (1994). "Experiences with Tripwire: Using Integrity Checkers for Intrusion Detection". Proceedings of the Third Annual System Administration, Networking and Security Conference (SANS III). p:89-101.

[9] Kim, G.H., Spafford, E.H. (1994). "The design and implementation of tripwire: a file system integrity checker". Proceedings of the 2nd ACM Conference on Computer and Communications Security. pp:18-29.

[10] Lai, C., Gong, L., Koved, L., Nadalin, A., and Schemers, R. (1999). "User Authentication and Authorization in the Java(tm) Platform". Proceedings of the 15th Annual Computer Security Applications Conference.

[11] Malabarba, S., Pandey, R., Gragg, J., Barr, E., and Barnes, J.F. (2000). "Runtime Support for Type-Safe Dynamic Java Classes". Lecture Notes in Computer Science, Vol. 1850. pp:337-361.

[12] Menezes, A., van Oorschot, P.C., and Vanstone, S.A. (1997). Handbook of Applied Cryptography. CRC Press.

[13] Qian, Z., Goldberg, A., and Coglio, A. (2000). "A Formal Specification of Java Class Loading”. Proceedings of OOPSLA'00. pp:325-336.

[14] Sterbenz, A. (1996). "An Evaluation of the Java Security Model". Proceedings of the 12th Annual Computer Security Applications Conference.

[15] Sun Microsystems (2001). "RMI Specification". http://java.sun.com/j2se/1.4/docs/ guide/rmi/spec/rmiTOC.html

[16] Sun Microsystems (2002a). "JPDA Enhancements in the JavaTM 2 SDK, v1.4" http://java.sun.com/j2se/1.4/docs/guide/jpda/enhancements.html\#hotswap

[17] Sun Microsystems (2002b). "Java 2 Platform, Standard Edition, v1.4.0 API Specification". http://java.sun.com/j2se/1.4/docs/api/index.html

[18] Wallach, D.S., and Felten, E.W. (1998). "Understanding Java Stack Inspection". Proceedings of the 1998 IEEE Symposium in Security and Privacy.

[19] Welch, L., and Stroud, R. (1999). "Supporting Real World Security Models in Java". Proceedings of the 7th IEEE Workshop on Future Trends of Distributed Computing Systems. 